\title{
Opinión de los vecinos sobre el uso de una finca adquirida por una universidad pública para fines socio-ambientales: el caso de Los Llanos, Costa Rica
}

\author{
Verónica Bonilla Villalobos \\ Maestría en Manejo de Recursos Naturales, UNED; veronicabonillav@gmail.com
}

Recibido 03-IV-2018 • Corregido 22-VI-2018 • Aceptado 10-VIII-2018

\begin{abstract}
Neighbors' opinion on the use of a farm acquired by a public university for socio-environmental purposes: the case of Los Llanos, Costa Rica. Social participation is a significant element in ecosystem restoration; and sustainability and acceptance depend on the participatory work of the surrounding communities. The principal goal of this study is to know neighbor opinion on the use of a 9 ha farm acquired by Universidad Estatal a Distancia (UNED) for socio-environmental purposes (Los Llanos, Alajuela, Costa Rica). I surveyed 41 of the 82 houses that adjoin the farm. $70 \%$ of the population has lived there for more than 15 years, only $4,3 \%$ finished university education; unemployment is $12 \%$ in men and $14 \%$ in women; around $9 \%$ lack access to drinking water. They reported that $54 \%$ use the farm for recreational and communal activities. Therefore, with adequate motivation, an additional third of the population could be incorporated into socio-environmental activities developed by the UNED, including restoration; and generating opportunities for work, training, entertainment, and reflection.
\end{abstract}

Key words: Los Llanos, ecological restoration, development socio-environmental, UNED, perception.
RESUMEN: La participación social es un elemento significativo en la restauración de los ecosistemas; la sostenibilidad y la aceptación dependen del trabajo participativo de las comunidades circundantes. El objetivo principal de este estudio es conocer la opinión de los vecinos sobre el uso de una hacienda de 9 ha adquirida por la Universidad Estatal a Distancia (UNED) con fines socioambientales (Los Llanos, Alajuela, Costa Rica). Inspeccioné 41 de las 82 casas que colindan con la granja. El 70\% de la población ha vivido allí durante más de 15 años, solo el 4,3\% terminó la educación universitaria; el desempleo es del $12 \%$ en los hombres y del $14 \%$ en las mujeres; alrededor del $9 \%$ no tiene acceso al agua potable. Informaron que el $54 \%$ usa la fincagranja para actividades recreativas y comunales. Por lo tanto, con una motivación adecuada, un tercio adicional de la población podría incorporarse a las actividades socioambientales desarrolladas por la UNED, incluida la restauración; y generar oportunidades de trabajo, capacitación, entretenimiento y reflexión.

Palabras clave: Los Llanos, restauración ecológica, desarrollo socio ambiental, UNED, percepción.
Los entornos sociales tienen una alta importancia para definir diversos procesos ambientales, entre los cuales se encuentra la restauración ecológica (Sinisterra, 2011; Maya, 2004; Rey Benayas, Newton, Diaz \& Bullock, 2009; Sánchez, 2001). Desde la perspectiva multidimensional de la percepción, se busca capturar una visión o pensamiento social de cómo se interpreta el mundo real, por medio de capturas subconscientes de las características de un determinado lugar o condiciones socioeconómicas y ambientales (Chardon, 1997). Dichas características buscan proporcionar información para evaluar los servicios ecosistémicos, ambientales y la participación comunitaria de los residentes de Los Llanos (Aguilar \& Ramírez, 2015).
La percepción social de una condición determinada no se interpreta de igual forma por cada individuo, esto debido a que cada persona dilucida de manera distinta los eventos y situaciones que se presentan alrededor (Maya, 2004). La percepción social es concebida como uno de los agentes de mayor importancia para la toma de decisiones en política pública, necesarios para la implementación de acciones y estrategias que garanticen las condiciones adecuadas para mejorar calidad de vida (Chardon, 1997; Lugo, Caicedo, Torres-Cuapa, Andrade \& Cruz, 2014).

Por lo anterior, es necesario establecer la pregunta: ¿cuál es el rol de la sociedad en el tema de la recuperación, 
control y protección de la biodiversidad para garantizar la sostenibilidad de los servicios ambientales de un ecosistema?, en especial, la finca Los Llanos y el impacto de la comunidad. La presente investigación analiza la percepción social de la comunidad de Los Llanos, respecto a los temas ambientales, económicos y sociales que giran en torno al proceso de toma de propiedad de la Universidad Estatal a Distancia (UNED) de Costa Rica sobre una finca con la que colinda esta comunidad. Este análisis se hace, ya que el éxito de proyectos futuros de restauración depende del involucramiento y participación de los miembros de la comunidad.

\section{METODOLOGÍA}

La investigación se desarrolló en la comunidad Los Llanos (durante el mes de julio de 2014), específicamente en una finca colindante de 9,6 ha, en la que los pobladores han llevado a cabo diversas actividades de aprovechamiento de servicios ecosistémicos, tales como recreación, captación de agua para consumo y aprovisionamiento. La comunidad y finca se localizan en el distrito La Garita, cantón Central de Alajuela, Costa Rica (9 $\left.58^{\prime} 44.94^{\prime \prime} \mathrm{N}-84^{\circ} 18^{\prime} 7.056^{\prime \prime} \mathrm{W}\right)$.
Esta finca fue donada a la Universidad Estatal a Distancia (UNED) por el Instituto Mixto de Ayuda Social (IMAS), en sesión 281-06-2013 del Consejo Directivo. La misma colinda al norte, este y oeste con una propiedad de la Hacienda Siquiares Ltda. y la Asociación de la Vivienda, Garita; al sur con la calle pública de Llanos y con la propiedad de Rafael Sandoval Brenes (IMAS, 2013) (Fig. 1). Por otra parte, la finca presenta un uso de suelo mayoritariamente conformado por pastizales con algunos relictos de vegetación arbórea nativa.

La historia de ocupación de la finca dicta una cobertura forestal muy fragmentada, con alto deterioro tanto a nivel florístico como de fauna y suelo, producto del uso extensivo de la ganadería y monocultivos que se presentó anteriormente en la propiedad (RECAS, 2014). Esto ocasionó un alto grado de degradación y pérdida de la cobertura boscosa. Es importante mencionar que la finca colinda con una naciente de agua, cuyo tanque de captación se encuentra dentro de la propiedad (RECAS, 2014) (Fig. 1).

El uso del suelo se ha mantenido por al menos 33 años, ya que el registro más antiguo identificado para la finca Los Llanos fue el plano de 1981. En él, se observa que la propiedad de Los Llanos estaba constituida por potrero, en su mayoría, y por caña de azúcar. Por la morfología
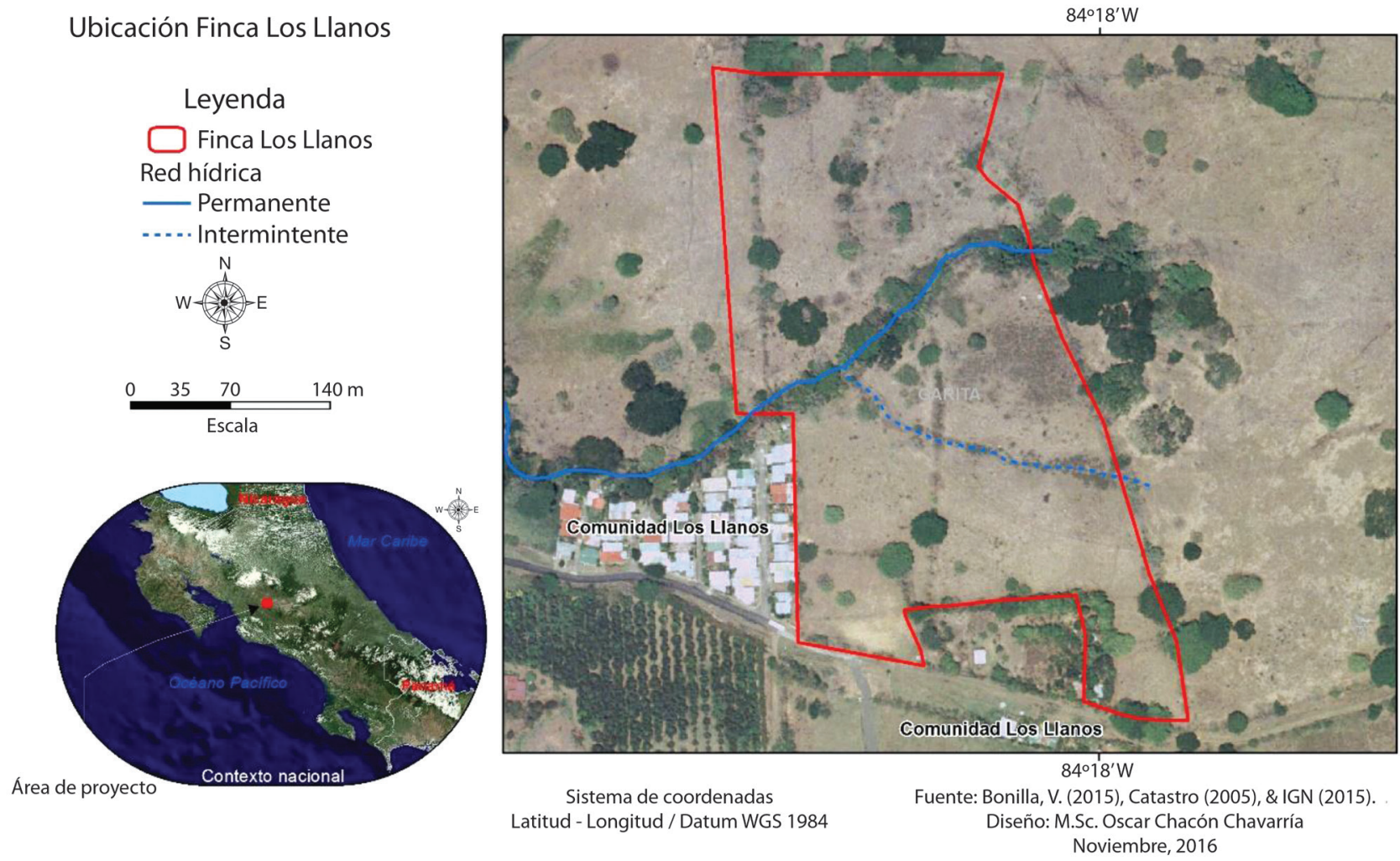

Fig. 1. Ubicación de la comunidad de Los Llanos y de la finca Los Llanos, la Garita; Alajuela, 2016. 
del suelo, solamente se pudo utilizar una sección para la construcción de viviendas y es en la que se encuentra actualmente la comunidad de Los Llanos.

La comunidad está conformada por un total de 82 viviendas, se encuentran distribuidas en dos bloques 0 sectores, uno con 68 viviendas colindantes con el sector oeste de la finca y otro de 14, colindantes con el sector sur. A partir del tamaño de la población residente en la comunidad, se definió una muestra al azar para ser entrevistada, para lo cual se aplicó la fórmula de Gómez (2008) y se obtuvo que se requería una muestra de 41 viviendas.

Posteriormente, se aplicó una encuesta con 43 preguntas 18 preguntas abiertas y 23 cerradas, estas se aplicaron a 26 mujeres y 15 hombres, mediante visitas en el sitio de residencia de los encuestados. Con el fin de garantizar un adecuado proceso de consulta, se hizo un esquema en el que se marcaron las casas encuestadas hasta cumplir con la muestra total. Los principales elementos incluidos en este instrumento estaban orientados a medir variables de índole social, ambiental y económica, como las siguientes:

- La caracterización de la población, la cual corresponde a datos personales para determinar desde hace cuánto tiempo viven en la zona e información para identificar fuentes de empleo y ocupación.

- Estructura poblacional según género, edad y nivel de escolaridad.

- Caracterización social, cultural y ambiental, la cual busca identificar el tipo de organización comunal, con el fin de determinar la mejor forma de llevar a cabo el proceso de construcción en retrospectiva de cómo era la finca Los Llanos, antes de su proceso de degradación. Por otra parte, se buscó determinar las actividades productivas, recreativas y educativas que se llevaban a cabo en la comunidad. También se intentó conocer el tipo de bosque y especies de árboles que existían en la finca, así como la fauna silvestre que había en el lugar antes de su proceso de degradación.
- Expectativas de uso de las instalaciones de la UNED, se identifican las expectativas que tienen sobre la participación de la UNED en la finca Los Llanos, qué problemática está presente, participación en proyectos comunales, perspectiva de la comunidad con proyectos sociales y de educación.

Este instrumento se aplicó a personas de ambos géneros, mayores de 18 años, sin límite máximo de edad, durante el mes de julio del 2014; las consultas se realizan sobre su entorno familiary algunas sobre sus condiciones.

\section{RESULTADOS}

Caracterización de la población: Se identificó que la mayoría de las familias encuestadas han vivido en la finca Los Llanos por más de 15 años (Fig. 2). Estas personas indican que su llegada fue mediante la Asociación Provivienda, la cual les facilitó el trámite de pago para adquirir los terrenos. Con ayuda del bono Costa RicaCanadá, lograron construir sus casas, la mayoría de las familias eran originalmente vecinas de la Garita de Alajuela y otras áreas aledañas.

La población de las viviendas entrevistadas se conformó por 88 mujeres (54\%), de las cuales 34 son amas de casa, 19 trabajan fuera, 12 no trabajan y 15 son estudiantes. En cuanto al género masculino, se contabilizaron 74 hombres (46\%), 47 trabajan fuera de la comunidad, nueve no cuentan con trabajo y 14 son estudiantes (Fig. 3). Por lo tanto, se evidencia una cantidad importante de mujeres que podrían involucrarse en actividades comunales y proyectos ambientales, así como un porcentaje más bajo de hombres.

Estructura poblacional según género, edad y nivel de escolaridad: se contabiliza un total de 162 habitantes, de los cuales el $54 \%$ son mujeres y el $46 \%$ son

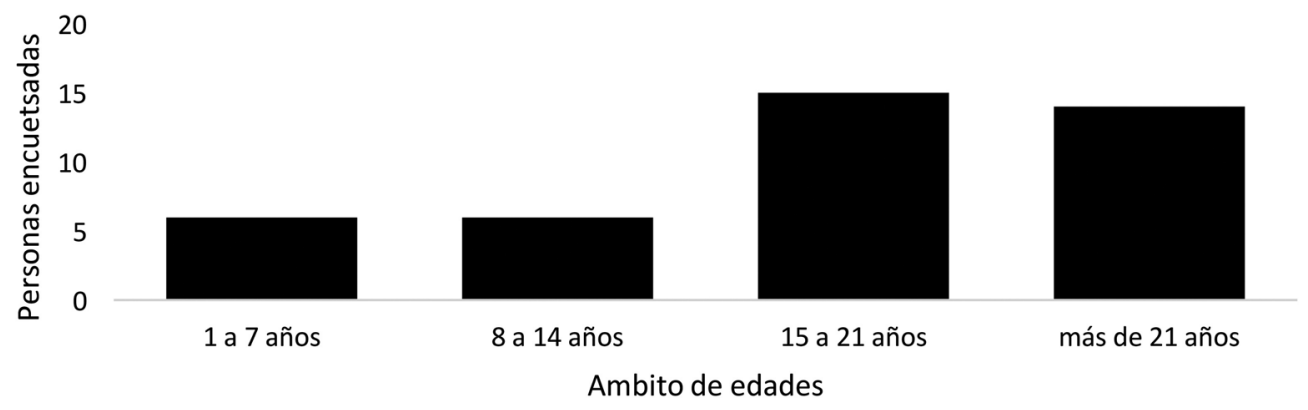

Fig. 2. Cantidad de años de residencia de las familias entrevistadas desde la constitución de la comunidad Los Llanos, La Garita, Alajuela, 2015. 


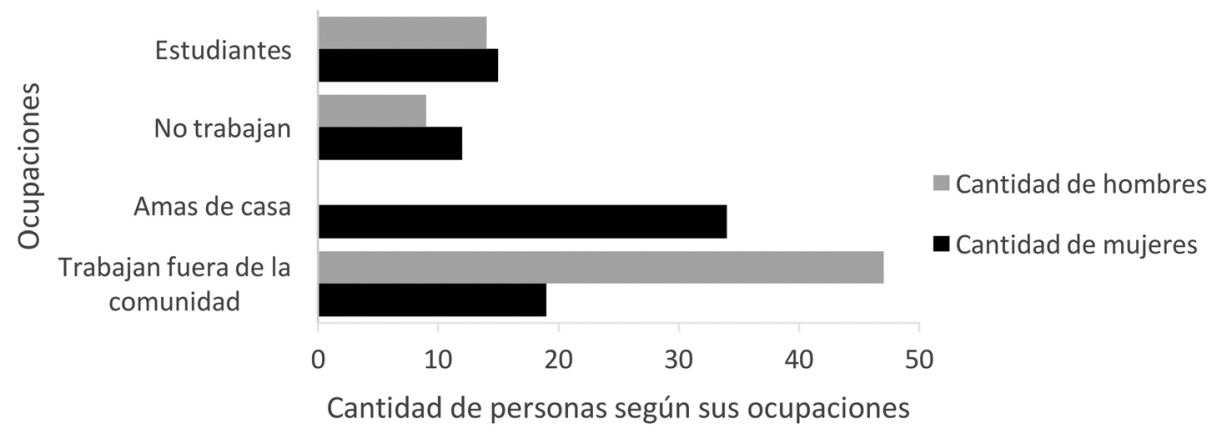

Fig. 3. Nivel de ocupación de la población entrevistada en Los Llanos, La Garita de Alajuela, 2015.

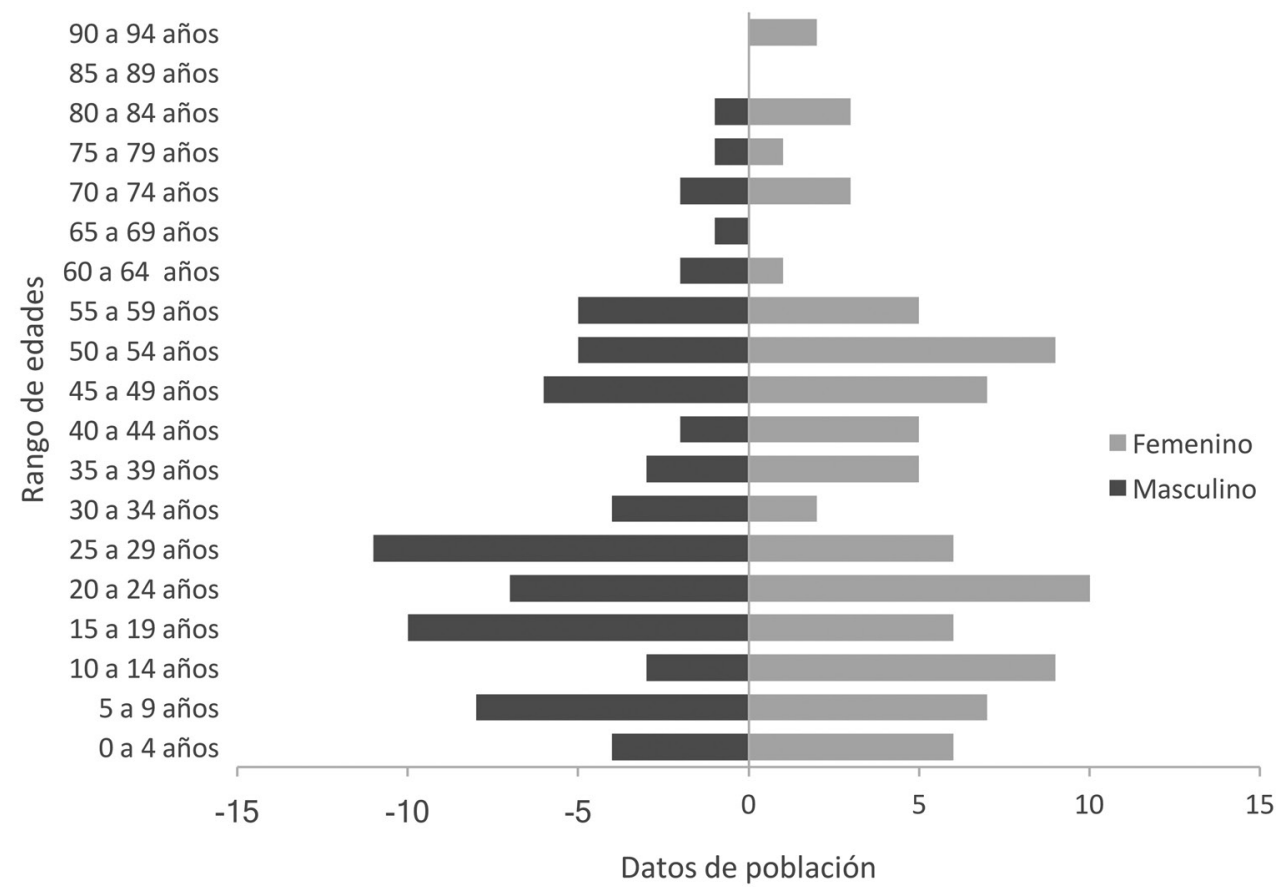

Fig. 4. Porcentaje de edades por rangos de los habitantes de la comunidad de Los Llanos, la Garita, Alajuela, 2014.

hombres. Las diferencias más importantes según sexo se presentan en el grupo de 10 a 14 años $(5,56 \%$ más mujeres), 25 a 29 años (6,79\% más hombres) y de 40 a 44 años (3,9 \% más mujeres). La mayor parte de los pobladores son jóvenes, con 25 años o menos (48,15\%), seguido de personas adultas entre 25 y 64 años $(43,21 \%)$, mientras que los adultos mayores representan el 8,64 \% (más de 65 años) (Fig. 4).

En cuanto a la escolaridad de las familias encuestadas, se identificó que el $34.6 \%$ tiene la primaria incompleta (el 12,97\% corresponde a estudiantes con edades de 5 a 12 años) y un $25.3 \%$ completa; tiene secundaria incompleta un $21.6 \%$ (donde un $8.02 \%$ corresponde a estudiantes de edades entre 13 y 18 años) y secundaria completa un $6.2 \%$; educación superior incompleta un
$1,2 \%$, un $4,3 \%$ educación superior completa y un $6.8 \%$ son infantes, con edades entre 1 y 5 años.

Asimismo, se correlaciona las diferentes ocupaciones, con respecto al grado de escolaridad, se obtiene tres principales: amas de casa con un $21 \%$, estudiantes con un $18 \%$ y dependientes con un $6 \%$. Se analiza el dato correspondiente amas de casa y el nivel de escolaridad que presentan, donde se identificó que el $41 \%$ de ellas tiene una primaria incompleta y el $30 \%$ primaria completa.

\section{Caracterización social, cultural y ambiental}

Residuos sólidos. Del total de encuestados, el $88 \%$ indicó no tener problemas con la recolección de basura 


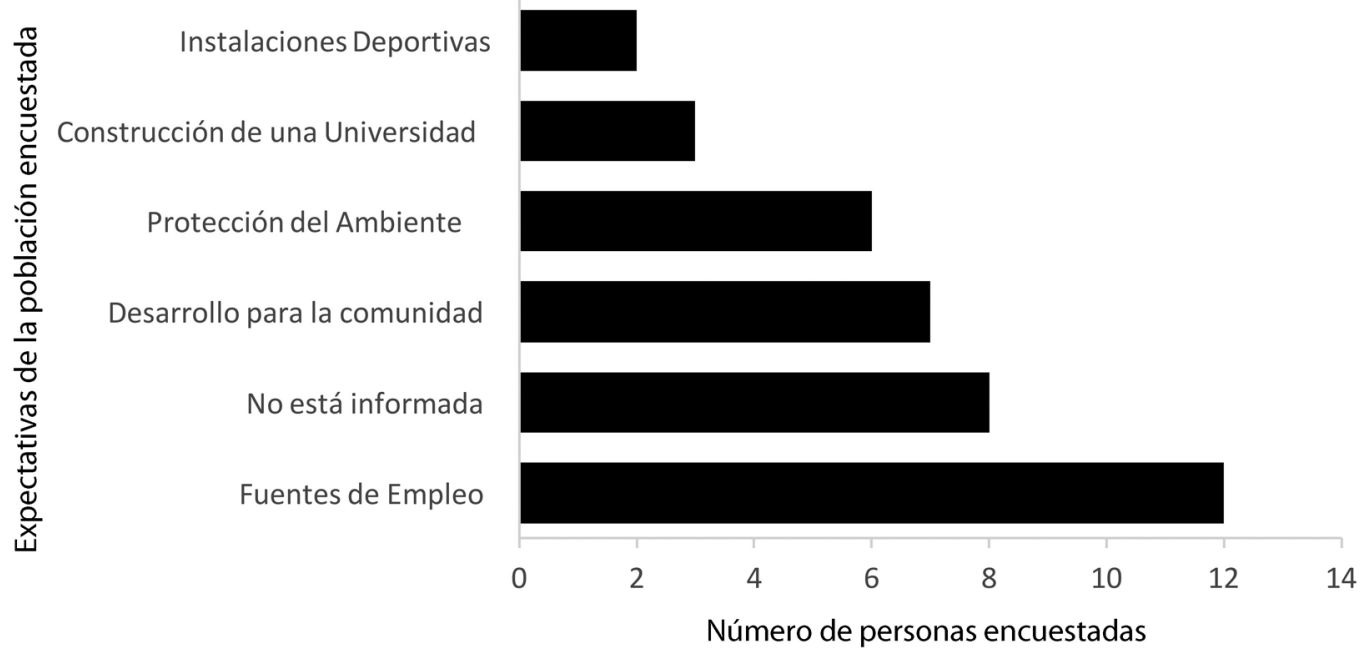

Fig. 5. Expectativas de la comunidad de Los Llanos, con la participación de la UNED en la finca Los Llanos, La Garita, Alajuela, 2015 ( $n=41)$.

y el $12 \%$ indicó que sí tiene problemas, debido a que el camión no llega hasta donde viven. En cuanto a la separación de estos residuos, el $54 \%$ no realiza separación de residuos sólidos, mientras el $46 \%$ indicó que sí los separan.

Aguas residuales. De los encuestados, el 34 \% indicó que las aguas residuales de sus viviendas son enviadas al río, sin importarles el impacto y el $66 \%$ indica que no. El sistema de distribución de aguas residuales carece de plantas de tratamiento, debido a que no existe un sistema de recolección de aguas.

Recurso hídrico. En cuanto al acceso al agua potable, el $91 \%$ de los encuestados cuenta con la disponibilidad de agua y el $9 \%$ restante indicó no contar con el servicio. Por otra parte, los encuestados mencionaron que tienen otros problemas, entre los cuales se encuentran la escasez (14\%) y otros conflictos que se dan por el recurso hídrico con la municipalidad y con personas ajenas a la comunidad que sacan agua sin permiso, entre otros.

Actividades productivas de la finca. Los entrevistados mencionan dos principales actividades de uso de la finca: una recreativa y otra productiva. Entre las actividades recreativas, el $54 \%$ mencionó utilizarla para fiestas familiares, reuniones comunales y partidos de fútbol. El $63 \%$ de los encuestados dijo que no utilizaba la finca para la actividad agrícola; en cuanto a la actividad ganadera, el $80 \%$ indicó no usarla, siendo parte esto del servicio ecosistémico de aprovisionamiento.

Se consultó sobre si recordaban alguna especie de flora y fauna en especial (árboles y animales silvestres) que hubiera y que ya no está presente en la finca, el $56 \%$ indica que no recuerda ninguna especie, el $39 \%$ indica que sí y un $5 \%$ dice que no sabe/ no responde. El $39 \%$ recuerda bosque en la finca y el $63 \%$ haber observado allí algún tipo de animal silvestre

Las especies de árboles recordadas por los encuestados fueron: Guanacaste (Enterolobium cyclocarpum), guapinol (Hymenaea courbaril), copey (Clusia major), laurel (Cordia alliodora), árboles frutales, higuerón (Ficus) y cedro (Cedrela spp.). Las especies de fauna más mencionadas fueron: conejos (Sylvilagus sp.), serpientes, muchas aves, garrobos (Ctenosaura similis), iguanas (Iguanidae), coyotes (Canis latrans), ardillas (Sciurus variegatus), roedores (Rodentia) y zorros (Didelphis marsupialis).

Expectativas de uso de las instalaciones de la finca Los Llanos. Los encuestados indicaron tener varias expectativas a raíz de la participación de la UNED en la finca Los Llanos, un 29 \% cree que generará fuentes de empleo (Fig. 5).

Así mismo, ante la pregunta: ¿les gustaría participar en algún grupo socioambiental u otro para trabajar con la comunidad? El 26,7 \% de los encuestados indicó que sí les gustaría participar (Fig. 6). 


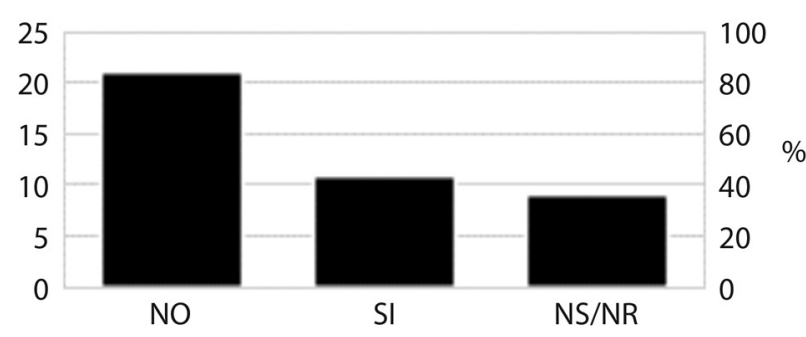

Fig. 6. Interés de participar en grupos comunitarios en la finca Los Llanos, La Garita, Alajuela, 2015. ( $n=41)$. Dónde: NS/NR (no sabe/no responde).

\section{DISCUSIÓN}

A través del diagnóstico social, se conoce la trayectoria histórica de la comunidad colindante con la finca Los Llanos, cómo está compuesta y sus necesidades inmediatas. Aronson et al. (2010) mencionan que es necesario establecer esta trayectoria, para así conocer cuál era el estado original de ese sitio y establecer una línea base para iniciar. Asimismo, caracterizar a la población es importante para el desarrollo de proyectos por parte de la UNED, como propulsora y promotora de la educación y posible gestora de proyectos en emprendedurismo (Lagunas, Beltrán, Urciaga \& Rubio, 2008).

Desde finales del siglo XX, hasta la década actual, diferentes estudios han señalado la importancia de la participación de las mujeres en el trabajo turístico comunitario, en espacios naturales y rurales (Lakovidou, 2002). En Los Llanos, se identificó un porcentaje importante de mujeres sin empleo remunerado que podrían participar en proyectos de carácter ambiental generados por la UNED. Esto fomentaría la participación y valoración de la mujer, además, promovería la sostenibilidad económica, social, cultural y ambiental, mediante la organización interna, la capacitación integral, la producción y la conservación de los recursos (Moreno-Casasola \& Salinas, 2007).

Dentro de la caracterización de la población, otro aspecto importante son los jóvenes, ya que, según Calle et al. (2008) la integración de estos en diversos procesos permite que desarrollen habilidades de indagación y generen soluciones adecuadas para su realidad biofísica y socioeconómica. En Los Llanos, se identificaron problemas que requieren una atención inmediata como la gestión de desechos sólidos, la contaminación de la quebrada con aguas residuales y el faltante del recurso hídrico. El aporte y participación de estos jóvenes podría brindar oportunidades y fortalecer la generación de líderes y actores comunales, además, del aprovechamiento del tiempo libre, que podría contribuir a la disminución de la violencia o el consumo de sustancias psicoactivas (Camacho-Ballestero, 2016).

Los servicios ecosistémicos que genera una finca son variados, entre ellos están los bienes o recursos naturales como el agua o los alimentos y la contribución de los ecosistemas a experiencias que benefician directa o indirectamente a las sociedades (Maass et al., 2005). Al respecto, se encontró que la comunidad de Los Llanos utiliza la propiedad para realizar actividades familiares, reuniones comunales, actividades recreativas como partidos de fútbol, así como actividades agrícolas y ganaderas, lo que genera un sentido de pertenencia por parte de ellos.

Por otro lado, se consulta a la comunidad encuestada si recuerdan especies de animales o árboles representativos de la finca, esto con el objetivo de crear una línea de las especies que se presentaban en el pasado y las actuales, para tener una visión general de las posibles especies ausentes, con el fin de contribuir a los proyectos de restauración ecológica. La participación social en las diferentes etapas de los procesos de restauración ecológica permite conocer el contexto de la comunidad, su posición y el componente social de su interés. Esto genera información acerca de las limitaciones y las oportunidades que hay en su entorno y que estos proyectos pueden fortalecer, generando nuevos líderes y la acción participativa (Sánchez, 2001; Maya, 2004; Rey et al., 2009; Sinisterra, 2011), tal como se muestra en los datos obtenidos.

Otros autores, como Sánchez, Márquez-Huitzil, Vega y Portales (2005), hacen referencia a la importancia de la interacción social entre los miembros de un colectivo y se complementa con la percepción de arraigo territorial y un sentimiento general de mutualidad e interdependencia; por consiguiente, en la comunidad de Los Llanos, hay familias que cuentan con más de 21 años de vivir allí, por lo tanto, ante estas dos aristas, se puede establecer una línea de seguimiento o trayectoria histórica de la comunidad, para crear enlaces en los procesos participativos.

Las iniciativas locales de restauración ecológica son una buena oportunidad para lograr que las comunidades adapten las herramientas de la investigación científica a su propio modo de ver el mundo y se conviertan en protagonistas de la recuperación de su entorno natural (Calle et al., 2008). Sin embargo, una de las respuestas que más llamó la atención fue la negativa de participar en actividades ambientales, no obstante, el $27 \%$ mostró interés, lo que indica que se deberá trabajar con herramientas atractivas y procesos inclusivos, para lograr la mayor participación e integración en el proceso de restauración ecológica (Aguilar \& Ramírez, 2015). 
Desde el punto de vista de la evaluación de los ecosistemas del milenio, la cual fue convocada por el secretario general de las Naciones Unidas en el año 2000 (Assessment, 2005), la finca Los Llanos presenta servicios ecosistémicos relacionados con los cuatro grandes grupos de servicios que proporcionan los ecosistemas, brindando oportunidades de uso directo e indirecto a la población vecina, a saber: 1) abastecimiento (acceso a un fuente directa de agua para las familias y las empresas; además, un recurso importante para establecer los proceso de restauración ecológica); 2) culturales (la comunidad utiliza la finca y el agua como fuentes de recreación familiar).

Por otra parte, el tercer punto se relaciona con la regulación, aportando beneficio a la población residente, gracias a la posibilidad de establecer un filtro en la carga de residuos químicos en los desechos líquidos, así como regulación del microclima local, estableciendo una mejora en la calidad del aire a partir de los procesos de restauración y 4) de apoyo (ofreciendo espacios de hábitat para las plantas y los animales, aportando una mejora en la diversidad de especies y manteniendo la diversidad genética, lo cual puede mejorar las condiciones necesarias para el establecimiento de polinizadores que permitan la mejora en los productos de cultivo y calidad genética de la flora de la región).

En síntesis, se requiere conocer las características de la comunidad en cuanto a su estructura poblacional, con el fin de identificar debilidades y fortalezas, para en un futuro integrar a las comunidades dentro de proyectos ambientales y dar respuesta a las políticas ambientales, sociales y educativas.

\section{AGRADECIMIENTOS}

Este diagnóstico fue realizado de forma colaborativa con estudiantes de la Maestría de Manejo de Recursos Naturales, liderados por las profesoras R. Ulate y $\mathrm{S}$. Avendaño. Este trabajo se realizó como parte de los requisitos para que la autora adquiera la Maestría en Manejo de Recursos Naturales de la Universidad Estatal a Distancia y optar por el grado de Magister Scientiae con énfasis en Biodiversidad. Wilmar Ovares Villalobos tradujo el resumen en inglés. Un especial agradecimiento a Johnny Villarreal Orias y Oscar Chacón Chavarría por sus aportes y comentarios.

\section{REFERENCIAS}

Aguilar, M., \& Ramírez, W. (2015). Monitoreo a procesos de restauración ecológica aplicado a ecosistemas terrestres; Monitoreo Participativo e Indicadores Socioeconómicos de la Restauración Ecológica, Calle, Carvajal \& Giraldo. Instituto de investigaciones de Recursos Biológicos von Humboldt, Bogotá, Colombia.

Aronson, J., Blignaut, J., Milton, J., Le Maitre, D., Esler, K. J., Limouzin, A., Fontaine, C., de Wit, M. P., Mugido, W., Prinsloo, P., van der Elst, L., \& Lederer, N. (2010). Are socioeconomic benefits of restoration adequately quantified? A meta-analysis of recent papers (2000- 2008) in Restoration Ecology and 12 other scientific journals. Restoration Ecology, 18(2), 143-154.

Assessment, M. E. (2005). Millennium ecosystem assessment. Ecosystems and human wellbeing: a framework for assessment Washington, DC: Island Press.

Calle, Z., Giraldo, E., Piedrahita, L., López, A., Cucuñame, J., Rivera, J. E., \& Caviche, R. (2008). Diálogo de saberes para la restauración ecológica de bosques: el papel de los niños y jóvenes investigadores. Revista Estudios Sociales Comparativos, 2, 68-85.

Camacho-Ballesteros, S. E. (2016). La restauración ecológica participativa: Una visión juvenil desde el territorio de Ciudad Bolívar. Revista Electrónica Educare, 20(2), 1-11

Gómez. (2008). Elementos de estadística descriptiva. San José, Costa Rica: EUNED.

Lagunas-Vázquez, M., Beltrán-Morales, L. F., Urciaga-García, J., \& Ortega-Rubio, A. (2008). Evaluación rural participativa: uso de los recursos naturales en la reserva de la biosfera. El Vizcaíno, BCS, México. Economía, sociedad y territorio, $8(26), 451-47$.

Lakovidou, O. (2002). Women's Agroturist Cooperatives in Greece: Key Elements for Their Successful Operation. Journal of Rural Cooperation, 30(1), 13-24.

Lugo-Morin, D. R., Caicedo, F., Torres-Cuapa, B., Andrade, J. C., \& Cruz, F. (2014). Cambio climático, género y percepción: Caso de la Laguna de Yahuarcocha. Spanish Journal of Rural Development, 5(4), 15-22

Maass, J., Balvanera, P., Castillo, A., Daily, G.C., Mooney, H.A., Ehrlich, P., Quesada, M., Miranda, A., Jaramillo, V.J., GarcíaOliva, F., Martínez-Yrizar, A., Cotler, H., López-Blanco, J., Pérez-Jiménez, A., Búrquez, A., Tinoco, C., Ceballos, G., Barraza, L., Ayala, R., \& Sarukhán, J. (2005). Ecosystem services of tropical dry forests: insights from longterm ecological and social research on the Pacific Coast of Mexico. Ecology and Society, 10(1), 17. Recuperado en http://www.ecologyandsociety.org/vol10/iss1/art17/ 
Maya, J, I. (2004). Sentido de comunidad y potenciación comunitaria. Apuntes de Psicología, 22(2), 187-211. Recuperado de http://personal.us.es/isidromj/php/wp-content/ uploads/2007/07/apuntes-sentido-de-comunidad.pdf.

Moreno-Casasola, P., \& Salinas, G. (2007). Programa de desarrollo comunitario sustentable y plan de manejo para la protección y conservación del Sitio Ramsar La Mancha-El Llano. Monografías Tercer Milenio, 6, 173185. Recuperado de http://sea-entomologia.org/PDF/ PDFSM3MVOL6/Pdf19173186019MorenoCasasola.pdf

RECAS (Red Ecología Ambiente y Sociedad). (2014). Propuesta de diseños de una estrategia de gestión para la recuperación ambiental y manejo ecológico de Finca Los Llanos. San José, Costa Rica: RECAS, UNED.
Rey Benayas, J. M., Newton, A. C., Diaz, A., \& Bullock, J. M. (2009). Enhancement of biodiversity and ecosystem services by ecological restoration: a meta-analysis. Science, 325, 1121-1124. doi: 10.1126/science.1172460.

Sánchez-Vidal, A. (2001). Medida y estructura interna del sentimiento de comunidad: un estudio empírico. Revista de Psicología Social, 16(2), 157-175.

Sinisterra, J. A., Calle, Z., Murgueitio, E., Sánchez, M., \& Rodríguez, G. (2011). Avances en la rehabilitación ecológica de la cárcava Monte Caldera, San Luis Potosí. In Vargas, O., \& Reyes, S. (eds.). La Restauración Ecológica en la Práctica: Memorias del I Congreso Colombiano de Restauración Ecológica y II Simposio Nacional de Experiencias en Restauración Ecológica. Bogotá D.C., Colombia: Universidad Nacional de Colombia. 